\title{
Title: Multispecies diel transcriptional oscillations in open ocean heterotrophic bacterial assemblages
}

\begin{abstract}
Authors: Elizabeth A. Ottesen ${ }^{1,2,3}$, Curtis R. Young ${ }^{1,2}$, Scott M. Gifford ${ }^{1,2}$, John M. Eppley, ${ }^{1,2}$, Roman Marin $\mathrm{III}^{4}$, Stephan C. Schuster ${ }^{5}$, Christopher A. Scholin ${ }^{4}$ and Edward F. DeLong ${ }^{1,2,6}$.
\end{abstract}

CONFIDENTIAL: Accepted, Science magazine, 5/22/2014:

\section{Affiliations:}

${ }^{1}$ Department of Civil and Environmental Engineering, Massachusetts Institute of Technology, Cambridge, MA 02139, USA.

${ }^{2}$ Center for Microbial Oceanography: Research and Education, University of Hawaii, Honolulu, HI 96822, USA.

${ }^{3}$ Department of Microbiology, University of Georgia, Athens, GA 30602, USA.

${ }^{4}$ Monterey Bay Aquarium Research Institute, Moss Landing, CA 95039, USA.

${ }^{5}$ Singapore Centre on Environmental Life Sciences Engineering, Nanyang Technological University, Singapore 637551.

${ }^{6}$ Department of Biological Engineering, Massachusetts Institute of Technology, Cambridge, MA 02139, USA.

*Correspondence to: delong@mit.edu; edelong@hawaii.edu 
21 Abstract: Oscillating diurnal rhythms of gene transcription, metabolic activity and behavior are

22 found in all three domains of life. Diel cycles in naturally occurring heterotrophic bacteria and

23 archaea however, have rarely been observed. Here we report time-resolved whole genome

24 transcriptome profiles of multiple, naturally occurring oceanic bacterial populations sampled in

25 situ over three days. As anticipated, the cyanobacterial transcriptome exhibited pronounced diel

26 periodicity. Unexpectedly however, several different heterotrophic bacterioplankton groups also

27 displayed diel cycling in many of their gene transcripts. Furthermore, diel oscillations in

28 different heterotrophic bacterial groups suggested population-specific timing of peak transcript

29 expression in a variety of metabolic gene suites. These staggered multispecies waves of diel gene

30 transcription may influence both the tempo and mode of matter and energy transformation in the

31 sea.

32

33 


\section{Main Text:}

The coordination of biological activities into daily periodic cycles is a common feature of eukaryotes and is widespread among plants, fungi, and animals, including man (1). Among single celled non-eukaryotic microbes, diel cycles have been well documented in cyanobacterial isolates (2-4), one halophilic archaeon (5), and in bacterial symbionts of fish and squid (6, 7). Some evidence for diel cycling in microbial plankton has also been suggested on the basis of bulk community amino acid incorporation, viral production, or metabolite consumption (8-10). The existence of regular diel oscillations in free-living heterotrophic bacterial species however, has rarely been assessed.

Microbial community RNA sequencing techniques now allow simultaneous determination of whole genome transcriptome profiles among multiple co-occurring species (11, 12), enabling high frequency, time resolved analyses of microbial community dynamics (12, 13). To better understand temporal transcriptional dynamics in oligotrophic bacterioplankton communities, we conducted a high-resolution multi-day time series of bacterioplankton sampled from the North Pacific Subtropical Gyre (14).

To facilitate repeated sampling of the same planktonic microbial populations through time, automated Lagrangian sampling of bacterioplankton was performed every two hours over three days using a free-drifting robotic Environmental Sample Processor (ESP; (13, 15); Fig. S1). Following instrument recovery, planktonic microbial RNA was extracted, purified and converted to cDNA to assess whole genome transcriptome dynamics of predominant planktonic microbial populations (Table S1, Table S2). The recovered cDNAs were dominated by transcripts from Prochlorococus and several proteorhodopsin-containing or photoheterotrophic bacteria, 
including members of the Pelagibacter (SAR11), Roseobacter, SAR116, SAR86, and SAR324 clades (Fig. S2).

Phylogenetic analysis of gene transcripts in the most abundant taxa revealed the presence of some microdiversity (Figs. S3-8). The most abundant transcripts sampled at any given time point however, were dominated by only a few genotypes within each population that persisted throughout the sampling period. An exception was Roseobacter, with transcripts for two different genes ( $g r o E L$ and $d n a K$ ) indicating the presence of a genotype that started at a very low abundance and increased in representation over the course of the time series. This variability could be due to an injection of a new population as water masses mixed during the latter portion of the time series, or possibly to an alteration in the relative transcriptional activities of two ecotypes that are responding to changes in the surrounding environment.

Transcriptional activity in Prochlorococcus was highly dependent on the time of day. Harmonic regression analyses indicated that nearly half $(1,491)$ of all Procholorococcus population transcripts were significantly periodic (Table 1; Table S3; Fig 1). The expression patterns observed were similar to those of monocultures growing in controlled laboratory settings (4) but there were also notable differences (Fig. 1). For example, photosystem I gene expression exhibited a double peak in the wild Prochlorococcus transcriptome around noon (Fig. 1). In contrast, under laboratory conditions most photosystem I genes, i.e. $p s a L$ and $p s a F$, were found to peak just before noon, while $p s a A$ and $p s a B$ peaked shortly after noon (4).

The largest discrepancy between Prochlorococcus laboratory studies and our field observations was that a considerable number of Prochlorococcus transcripts in our field populations peaked around midday (Fig. 1). Some of these genes did exhibit periodicity in cultures, but peaked at a different time of day in field populations. A larger fraction of these 
mid-day peaking transcripts were either not periodically expressed, or were not present in the culture experiments. In addition, $62 \%$ of the $10 \mathrm{am}-4 \mathrm{pm}$ peaking transcripts in our field study lacked KEGG orthology annotations, as opposed to those peaking in the evening or late at night.

A number of factors may be responsible for differences in transcript dynamics between in laboratory cultures versus field Prochlorococcus populations. Maximal light levels at our study site at $23 \mathrm{~m}$ depth were frequently two fold higher $\left(450 \mathrm{umol} \mathrm{Q} / \mathrm{m}^{-2} / \mathrm{s}^{-1}\right)$ than those used in laboratory microarray experiments $\left(232 \mathrm{umol} \mathrm{Q} / \mathrm{m}^{-2} / \mathrm{s}^{-1}\right)(4)$. Fundamental genetic differences between our field populations and the Prochlorococcus strain used in laboratory culture experiments likely also contribute to the differences we observed. Other variables, including nutrient composition and organismal interactions, may also be a factor in the observed differences. While we could not identify obvious trends in the type or function of transcripts showing peak expression during the mid-day period, they did include a wide range of enzymatic functions that are more consistent with nutrient-responsive metabolic changes rather than a simple high-light stress response.

An abundant Roseobacter population also showed strong diel oscillations in its transcriptome profile, most notably in expressed genes involved in bacteriochlorophyllassociated aerobic anoxygenic photosynthesis (AAnP). Overall, a large fraction of Roseobacter transcripts were periodically expressed (Table 1). Of these, the majority peaked during daylight hours, with only a few gene transcripts peaking at night (Fig. 2). While this pattern contrasts with that observed in Prochlorococcus, where most diurnally regulated transcripts peaked at dawn or dusk, it was consistent with transcriptional regulation recently reported in Dinoroseobacter shibae (16). 
102

103

104

105

106

107

108

109

110

111

112

113

114

115

116

117

118

119

120

121

122

123

$11 \mathrm{pm}$ and 7 am encoded genes belonging to a large photosynthetic "superoperon" (Fig. S9).

Nightly expression of these genes, followed by immediate repression upon light onset, is consistent with the D. shibae study (16), and may be preparing cells for efficient solar energy harvest in the early morning hours. Functions that peaked during the day-time hours included ribosomal proteins, respiratory transcripts, genes involved in amino acid metabolism, and transporters (Fig. 2).

Proteorhodopsin-containing photoheterotrophs including members of the SAR11, SAR116, and SAR86 also showed evidence of diel periodicity in many of their gene transcripts (Fig. 2, Table 1). Interestingly, all opsin-containing bacteria analyzed (SAR11, SAR116, SAR86, and SAR324) exhibited statistically significant diel oscillations in their proteorhodopsin gene transcripts (Table S3; Fig. S10). Peak expression of the opsin transcripts occurred near dawn in all these populations (Fig. S10), potentially optimizing solar energy capture by the light-driven, proton-pumping rhodopsins.

Principal components analysis distinguished time series samples for each heterotroph by time of day (Fig. 3) and showed significant correlation with the light-driven behavior of Prochlorococcus (Table 1). Overall, this data is consistent with profound, genome-wide transcriptional changes across the day-night cycle for each population. In addition, co-clustering of transcripts using GeneARMA $(\mathrm{GA} ;(14,17))$ revealed suites of gene transcripts that exhibited similar expression patterns among different taxa (Fig. 4; Fig. S11-14; Table S4). For example, a group of transcripts that that fit highly similar GA expression models across multiple species included Pro GA5, Pro GA7, Pro GA9, Pro GA23, SAR11 GA6. SAR11 GA18, SAR116 GA2, and Roseobacter GA8 (Fig 4; Fig S14; Table S4). These multispecies, day-peaking transcripts 
124 (Fig S14; Table S4) included gene products associated with respiration (Procholorococcus, 125 SAR11, SAR116, Roseobacter), nitrogen metabolism (Procholorococcus, SAR11, SAR116), 126 glycine metabolism (Procholorococcus, SAR11, Roseobacter), carbon monoxide metabolism 127 (SAR116, Roseobacter) and DNA synthesis (Procholorococcus, Roseobacter). This coclustering of gene transcripts reveals a complex pattern of expression through the day and across the time series, and provides evidence for parallel trends in gene expression across multiple species (Fig. 4, Fig. S11-14, Table S4).

Together, the transcriptional profiles of Roseobacter, SAR11, SAR116 and SAR86 indicate diel cycling of metabolic gene transcripts, and suggest a multispecies wave-like progression of upregulated gene suites across the day/night cycle (Fig. 4). Most conspicuously, a regular diel succession of translational, transcriptional and respiratory gene transcripts was followed by peaks in transporter transcripts that possibly reflect a metabolic recovery phase (Fig. 2). Many of these metabolic pathway transcripts peaked earlier in the day in Roseobacter field populations relative to other bacterial heterotrophs (Fig. 2, Fig 4, Table S4).

The overall transcriptional profile of SAR324 did not show as many transcript diel oscillations as other heterotrophic taxa. Instead, principal components analysis clustered SAR324 transcripts according to the day that they were collected (Fig. 3). In particular, the SAR324 group showed a strong separation between the first portion of the time series and the second in principal components analysis (Fig. 3). This split appears to be associated with the increases in temperature and salinity observed across the time series (Fig. S1).

The diurnal patterns reported here for open ocean heterotrophic bacterioplankton were different from those observed in a previous study of phylogenetically related coastal bacterioplankton using similar methods (12). For example, coastal versus open ocean SAR11 
147 populations revealed differential expression levels among several orthologous transcript categories (Fig. S15). Additionally, while the open ocean SAR11 populations reported here exhibited statistically significant diel oscillations for many gene transcripts (Fig. 2), the coastal SAR11 populations did not.

Currently available data are insufficient to provide definitive mechanistic explanations for the diel behaviors we observed in different heterotrophic bacterioplankton species. It is possible that photoreceptors in these bacteria are involved in regulating light-dark cycles of transcriptional activity. Marine Roseobacter species have previously been shown to regulate their global transcriptional behavior in response to light (16), and laboratory cultures of Pelagibacter also exhibit light-responsive metabolic behaviors (18). Differences between the behaviors of SAR11 coastal versus open ocean field populations however (Fig. S15), as well as comparisons of several taxa in our field study versus laboratory experiments on related cultivated isolates (Fig. 1), suggest that other factors may be at play in regulating diel behavior among these different bacterioplankton populations.

Previous studies have proposed that tight metabolic coupling between primary producers and consumers in microbial plankton might elicit conspicuous diel cycling in heterotrophic bacterial activities (8). The diel cycling we observed among different bacterioplankton species is consistent with this hypothesis, with multiple co-existing heterotroph populations exhibiting diurnal oscillations resembling those of their photoautotrophic neighbors. We postulate that the tightly coupled multispecies temporal expression patterns observed may elicit corresponding waves of species-specific metabolic responses at regular time intervals, potentially coordinating diverse biogeochemical activities in these complex microbial communities. Such temporal coordination of biogeochemical activities among multiple species may be important regulators of 
both the tempo and mode of microbial matter and energy transformation in the sea.

171

172

173

174

175

176

177

178

179

180

181

182

183

184

185

186

187

188

189

190

191

192

193

194

195

196

197

198

199

200

201

202

203

204

205

206

207

208

209

210

211

212

213

214

215

216

217

218

219

220

\section{References and Notes:}

1. D. Bell-Pedersen et al., Circadian rhythms from multiple oscillators: lessons from diverse organisms. Nature reviews. Genetics 6, 544 (2005).

2. I. M. Axmann, S. Hertel, A. Wiegard, A. K. Dorrich, A. Wilde, Diversity of KaiC-based timing systems in marine Cyanobacteria. Marine genomics In press, http://dx.doi.org/10.1016/j.margen.2013.12.006, (2014).

3. H. Ito et al., Cyanobacterial daily life with Kai-based circadian and diurnal genome-wide transcriptional control in Synechococcus elongatus. Proc. Natl. Acad. Sci. U.S.A. 106, 14168 (2009).

4. E. R. Zinser et al., Choreography of the transcriptome, photophysiology, and cell cycle of a minimal photoautotroph, prochlorococcus. PloS one 4, e5135 (2009).

5. K. Whitehead, M. Pan, K. Masumura, R. Bonneau, N. S. Baliga, Diurnally entrained anticipatory behavior in archaea. PloS one 4, e5485 (2009).

6. J. F. Flint, D. Drzymalski, W. L. Montgomery, G. Southam, E. R. Angert, Nocturnal production of endospores in natural populations of Epulopiscium-like surgeonfish symbionts. Journal of bacteriology 187, 7460 (2005).

7. A. M. Wier et al., Transcriptional patterns in both host and bacterium underlie a daily rhythm of anatomical and metabolic change in a beneficial symbiosis. Proc. Natl. Acad. Sci. U.S.A. 107, 2259 (2010).

8. J. M. Gasol et al., Diel variations in bacterial heterotrophic activity and growth in the northwestern Mediterranean Sea. Mar Ecol Prog Ser 164, 107 (1998).

9. C. Winter, G. J. Herndl, M. G. Weinbauer, Diel cycles in viral infection of bacterioplankton in the North Sea. Aquat Microb Ecol 35, 207 (2004).

10. M. Galí et al., Diel patterns of oceanic dimethylsulfide (DMS) cycling: Microbial and physical drivers. Global Biogeochem Cycles 27, 620 (2013).

11. S. M. Gifford, S. Sharma, M. Booth, M. A. Moran, Expression patterns reveal niche diversification in a marine microbial assemblage. The ISME journal 7, 281 (2013).

12. E. A. Ottesen et al., Pattern and synchrony of gene expression among sympatric marine microbial populations. Proc. Natl. Acad. Sci. U.S.A. 110, E488 (2013).

13. E. A. Ottesen et al., Metatranscriptomic analysis of autonomously collected and preserved marine bacterioplankton. The ISME journal 5, 1881 (2011).

14. Materials and methods are available as supplementary material on Science Online

15. C. M. Preston et al., Underwater application of quantitative PCR on an ocean mooring. PloS one 6, e22522 (2011).

16. J. Tomasch, R. Gohl, B. Bunk, M. S. Diez, I. Wagner-Döbler, Transcriptional response of the photoheterotrophic marine bacterium Dinoroseobacter shibae to changing light regimes. The ISME journal 5, 1957 (2011).

17. N. Li et al., Functional clustering of periodic transcriptional profiles through $\operatorname{ARMA}(p, q)$. PloS one 5, e9894 (2010).

18. L. Steindler, M. S. Schwalbach, D. P. Smith, F. Chan, S. J. Giovannoni, Energy starved Candidatus Pelagibacter ubique substitutes light-mediated ATP production for endogenous carbon respiration. PloS one 6, e19725 (2011).

19. Z. Liu, W. Hsiao, B. L. Cantarel, E. F. Drábek, C. Fraser-Liggett, Sparse distance-based learning for simultaneous multiclass classification and feature selection of metagenomic data. Bioinformatics 27, 3242 (2011).

20. J. Okansen et al. Package "vegan": Community ecology package. http://cran.rproject.org/web/packages/vegan//vegan.pdf (2012).

21. J. C. Robidart et al., Ecogenomic sensor reveals controls on N-fixing microorganisms in the North Pacific Ocean. The ISME journal doi: 10.1038/ismej.2013.244, (2014).

22. F. J. Stewart, E. A. Ottesen, E. F. DeLong, Development and quantitative analyses of a universal rRNAsubtraction protocol for microbial metatranscriptomics. The ISME journal 4, 896 (2010). 
23. Y. Shi, G. W. Tyson, J. M. Eppley, E. F. DeLong, Integrated metatranscriptomic and metagenomic analyses of stratified microbial assemblages in the open ocean. The ISME journal 5, 999 (2011).

24. S. Rodrigue et al., Unlocking short read sequencing for metagenomics. PloS one 5, e11840 (2010).

25. S. M. Kielbasa, R. Wan, K. Sato, P. Horton, M. C. Frith, Adaptive seeds tame genomic sequence comparison. Genome research 21, 487 (2011).

26. B. K. Swan et al., Prevalent genome streamlining and latitudinal divergence of planktonic bacteria in the surface ocean. Proc. Natl. Acad. Sci. U.S.A. 110, 11463 (2013).

27. R. J. Newton et al., Genome characteristics of a generalist marine bacterial lineage. The ISME journal 4, 784 (2010).

28. M. Kanehisa, S. Goto, KEGG: Kyoto encyclopedia of genes and genomes. Nucleic Acids Res. 28, 27 (2000).

29. Team RDC (2012) R: A Language and Environment for Statistical Computing (R Foundation for Statistical Computing, Vienna).

30. Y. Benjamini, Y. Hochberg, Controlling the false discovery rate - a practical and powerful approach to multiple testing. J Roy Stat Soc B Met 57, 289 (1995).

31. J. P. Baudry, A. E. Raftery, G. Celeux, K. Lo, R. Gottardo, Combining Mixture Components for Clustering. Journal of computational and graphical statistics : a joint publication of American Statistical Association, Institute of Mathematical Statistics, Interface Foundation of North America 9, 332 (2010).

32. R. C. Edgar, Search and clustering orders of magnitude faster than BLAST. Bioinformatics 26, 2460 (2010).

33. R. C. Edgar, MUSCLE: multiple sequence alignment with high accuracy and high throughput. Nucleic Acids Res. 32, 1792 (2004).

34. M. N. Price, P. S. Dehal, A. P. Arkin, FastTree: computing large minimum evolution trees with profiles instead of a distance matrix. Molecular biology and evolution 26, 1641 (2009).

35. J. G. Caporaso et al., QIIME allows analysis of high-throughput community sequencing data. Nature methods 7, 335 (2010).

\section{Acknowledgments:}

We thank the officers and crew of the Kilo Moana, Chief Scientist Julie Robidart, Sam Wilson, and the ESP engineering and Science team (John Ryan, Jim Birch, Chris Preston, Gene Massion, Scott Jensen, Brent Roman) for all the able assistance. This work was supported by grants from the Gordon and Betty Moore Foundation GBMF \#492.01 and \#3777 (EFD) and NSF grant EF0424599 (EFD). Development of the ESP was supported by National Science Foundation Grant OCE-0314222 (to C.A.S.), National Aeronautics and Space Administration Astrobiology Grants NNG06GB34G and NNX09AB78G (to C.A.S.), the Gordon and Betty Moore Foundation (C.A.S.), and the David and Lucile Packard Foundation. This work is a contribution of the Center for Microbial Oceanography: Research and Education (C-MORE). Sequences reported in 
259 this paper have been deposited in the Gen-Bank database (accession no. SRP041215).

260

261 


\section{Supplementary Materials:}

Materials and Methods

Figures S1-S14

Table S1

Legends for External Tables S2-S4

References (27-43)

Fig. 1. Laboratory versus field comparisons of periodic expression patterns in Prochlorococcus populations. A. Scatter plot shows time of peak abundance for 973 transcripts identified as significantly periodic in both studies. Histograms show the total number of genes peaking in 1hour intervals in this study (top) and the laboratory experiment (side). Black bars represent genes identified as significantly periodic in both studies, grey bars represent genes expressed in both studies but significantly periodic in only one, and white bars represent significantly periodic transcripts that were not detected in the other dataset. For this comparison, we used published significance cutoffs from the laboratory study (4), but for consistency generated new peak times using our harmonic regression approach and the published normalized mean expression levels for each time point. In general, the peak times generated using our approach closely matched published values for that dataset. B-C. Plots showing relative expression (normalized to mean expression level) over time for our metatranscriptome (top trace) and in microarray data (bottom trace) for selected transcripts. For comparison, experimental midnights ( $24 \mathrm{hr}$ and $48 \mathrm{hr}$ ) from the microarray study are aligned with the 12:00AM samples from 9/9 and 9/10, respectively. All ATP synthase subunits (B) and selected subunits from photosystem I (C) are shown.

Fig. 2. Timing of periodically expressed transcripts. For each population, a histogram showing the number of periodically expressed diel transcripts with peak expression within 1-hr intervals throughout the day is shown (left). On the right, time of peak expression of all transcripts assigned to selected KEGG pathways is plotted (grey). Red circles denote transcripts identified as significantly periodic ( 24 hour period). The "transporters" category includes both the ABC Transporters KEGG pathway and the Transporters BRITE hierarchy, the "photosynthesis" category includes both the Photosynthesis KEGG pathway and the BRITE Photosynthesis Proteins categorizations. "Carbon Fixation" refers to genes assigned to the Carbon Fixation in Photosynthetic Organisms KEGG Pathway. The photosynthesis and carbon fixation categories are present in heterotrophic organisms due to cross-assignment of ATP Synthase genes and pentose phosphate cycle genes. Black and yellow bars depict the daily photoperiod (based on sunrise and sunset times).

Fig. 3. Principal Component analysis of population transcriptional profiles. Transcript abundances were normalized to total transcripts assigned to each population at each time point, 
301 and arcsin transformed to approximate normality (19). Symbol color denotes time of day and 302 shape denotes day of collection. Grey lines connect samples to centroids for selected sample 303 groupings that separate points well. Roseobacter SAGs: samples collected between 7am and 9pm 304 (vs. 9pm to 7am); SAR11, SAR116 and SAR86 cluster, samples collected between 9am and 6pm 305 (and vice versa); SAR324 cluster, samples collected before or after 9/9 4pm. All factor 306 correlations shown were highly significant $(\mathrm{p}=0.001)$. Alternative time of day categories were 307 also highly significant for Roseobacter SAGs, SAR11, SAR116 and SAR86. SAR116 $\left(\mathrm{r}^{2} 0.10, \mathrm{p}\right.$ 308 0.037) and SAR86 ( $\mathrm{r}^{2}$ 0.12, p 0.019) also correlated weakly with the grouping shown for 309 SAR324. All analyses carried out using functions in the vegan software package (20).

311 Fig. 4. Timing of expression of functional gene clusters in different taxa clustered by similarity. 312 Heatmap shows cluster models for all geneARMA clusters, colored by mean-centered relative 313 expression (red=high, blue=low). Black and yellow bars show the daily photoperiod. Each box 314 represents a single sampling event, for sample times see Table S1. Dendrograms show cluster 315 model similarity (Pearson correlations, average linkage clustering, scale bar at upper right 316 represents a correlation of 0.5 ). The total number of genes (A), significantly periodic genes (B), 317 and genes associated with Photosynthesis (C), Ribosome (D), Oxidative Phosphoryloation (E), 318 Amino Acid Metabolism (F), and Transport (G) (defined as for Figure 2), are listed for each 319 cluster. 
Table 1: Harmonic Regression Results

\begin{tabular}{lcccccc}
\hline & Prochlorococcus & $\begin{array}{c}\text { Roseobacter } \\
\text { SAGs }\end{array}$ & SAR11 & SAR116 & SAR86 & SAR324 \\
\hline Sequence reads $^{1}$ & 2886677 & 177982 & 774064 & 200368 & 151468 & 118098 \\
Transcripts $^{2}$ & 3045 & 2604 & 2802 & 2618 & 2367 & 4732 \\
Periodic $^{3}$ & 1491 & 426 & 201 & 80 & 10 & 8 \\
Constrained PCA vs $^{24-h o u r ~ c l o c k ~}$ & 0.68 & 0.49 & 0.24 & 0.15 & 0.13 & 0.10 \\
24-cis & $(\mathrm{p}=0.005)$ & $(\mathrm{p}=0.005)$ & $(\mathrm{p}=0.005)$ & $(\mathrm{p}=0.005)$ & $(\mathrm{p}=0.005)$ & $(\mathrm{p}=0.01)$ \\
Procrustes Test vs. & & 0.78 & 0.55 & 0.70 & 0.52 & 0.36 \\
Prochlorococcus $^{\text {PCA }}$ & & $(\mathrm{p}<0.001)$ & $(\mathrm{p}<0.001)$ & $(\mathrm{p}<0.001)$ & $(\mathrm{p}<0.001)$ & $(\mathrm{p}=0.031)$ \\
Mantel Test vs. $_{\text {Prochlorococcus }^{6}}$ & & 0.63 & 0.40 & 0.31 & 0.26 & 0.27 \\
\hline
\end{tabular}

The total number of sequence reads assigned to each taxon bin

${ }^{2}$ The total number of unique ortholog clusters (see Database S1) with at least one mapped sequence.

${ }^{3}$ The total number of sequences identified as showing 24-hour periodicity using harmonic regression.

${ }^{4}$ Proportion of variance explained by 24 -hour periodicity in constrained principal components analysis.

5 Procrustes correlation between the first two principal components from Prochlorococcus and other taxa (unconstrained principal components analysis as shown in Fig. 3). P-value based on 999 permutations.

${ }^{6}$ Correlation between pairwise sample similarities from Prochlorococcus and heterotrophic taxa based on Mantel test on Euclidean distance matrices. P-value based on 999 permutations. 


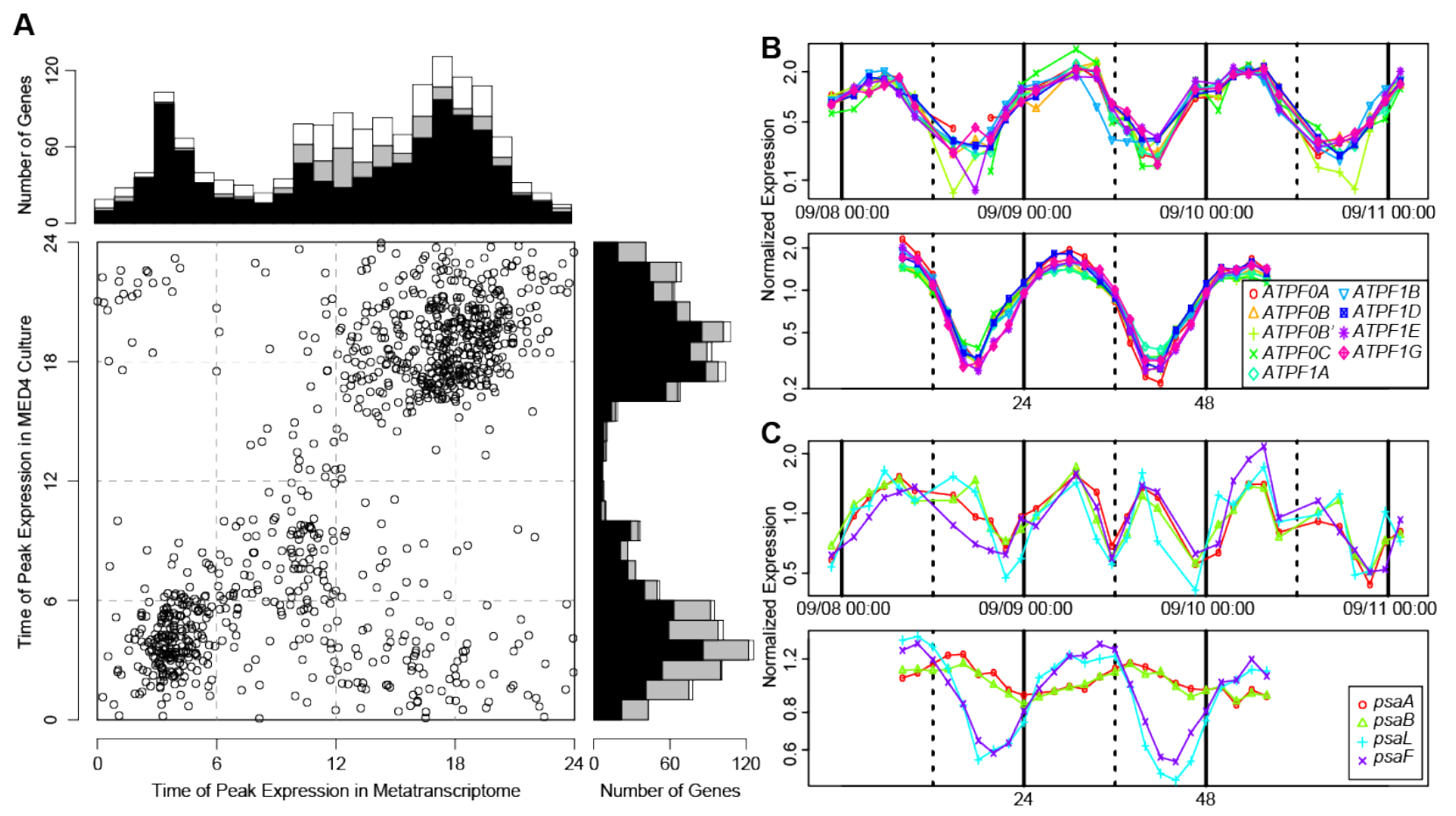

15 

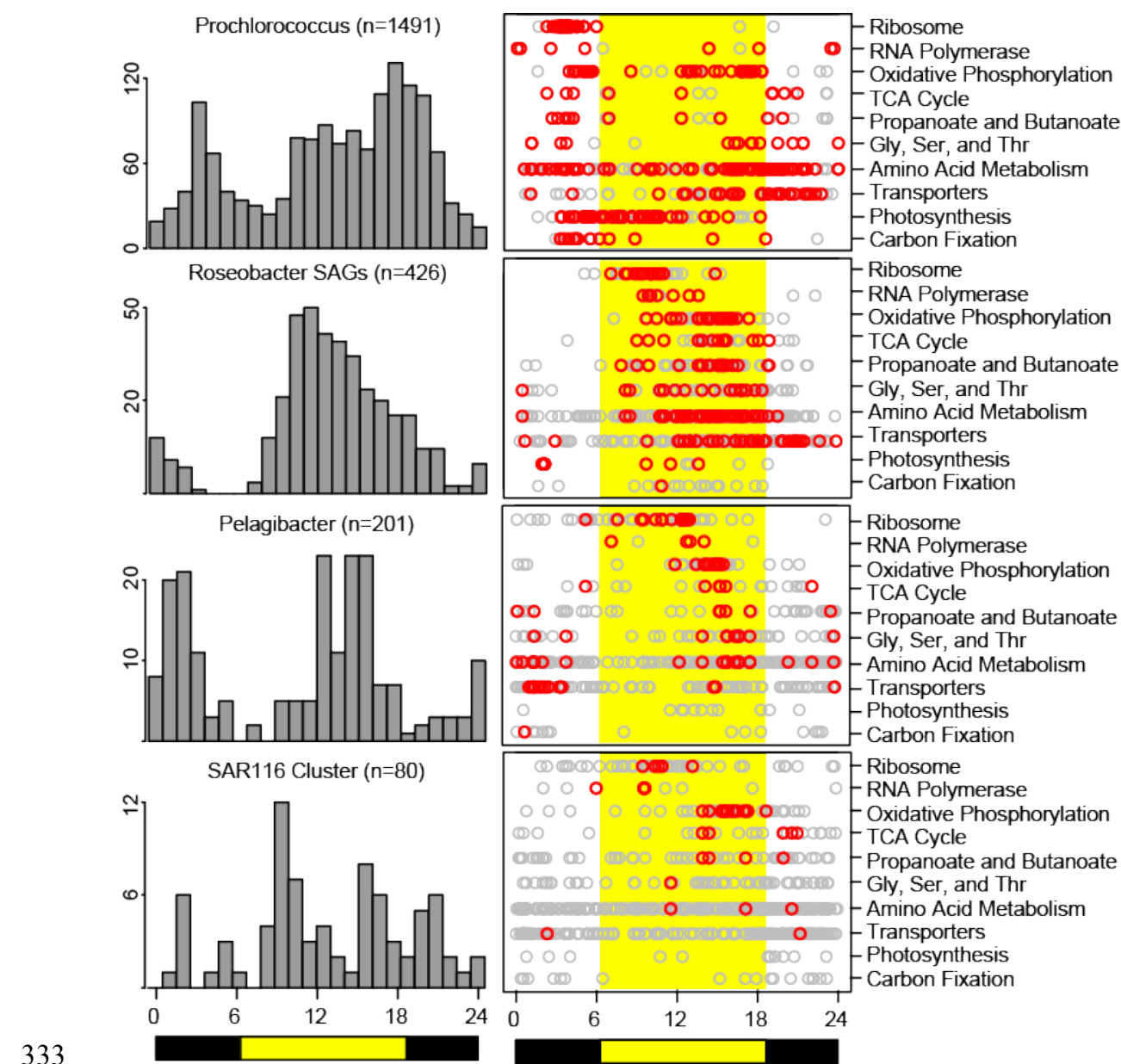

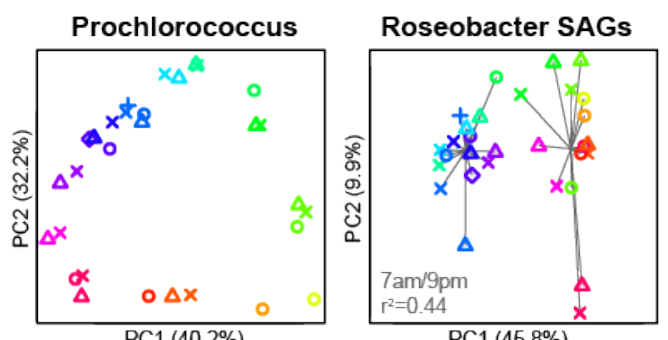

Pelagibacter

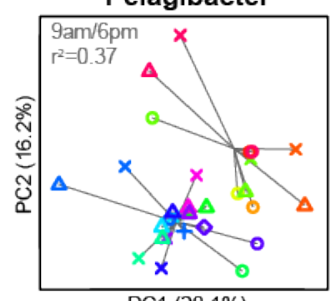

PC1 (28.1\%) SAR86 cluster

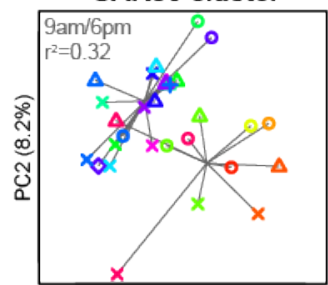

PC1 (13.3\%)

SAR116 cluster

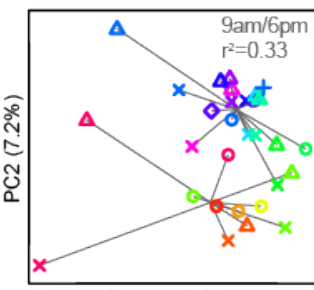

PC1 (16.4\%)

SAR324 cluster

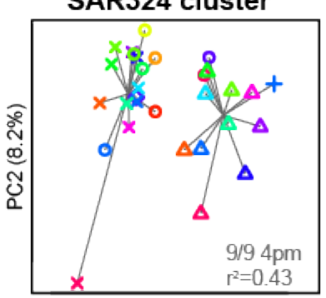

9/7 10:38pm

$\times$ 9/8 1:37am

$\times 9 / 8$ 3.36am

$\times 9 / 85.36 a m$

$\times$ 9/8 7:35am

$\times 9 / 8241 \mathrm{pm}$

$\times$ 9/8 5:36pm $\times 9 / 87.37 \mathrm{pm}$ $\times 9 / 89.36 \mathrm{pm}$

$\times$ 9/8 11:37pm

- 9/9 1:36am

- 9/9 6:53am

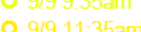

- $9 / 91: 35 \mathrm{pm}$

- 9/9 3:34pm

○ 9/9 5:36pm

- 9/9 10:36pm

$\Delta$ 9/10 1:37am

$\Delta$ 9/10 3:36am

$\Delta$ 9/10 5:36am

$\Delta$ 9/10 7:37am

$\Delta$ 9/10 2-46pm

$\Delta$ 9/10 5:36pm

$\Delta$ 9/10 7:36pm

$\Delta$ 9/10 9:35pm

$\Delta$ 9/10 11:36pm

$+9 / 11$ 1:36am

PC1 (12.1\%) 


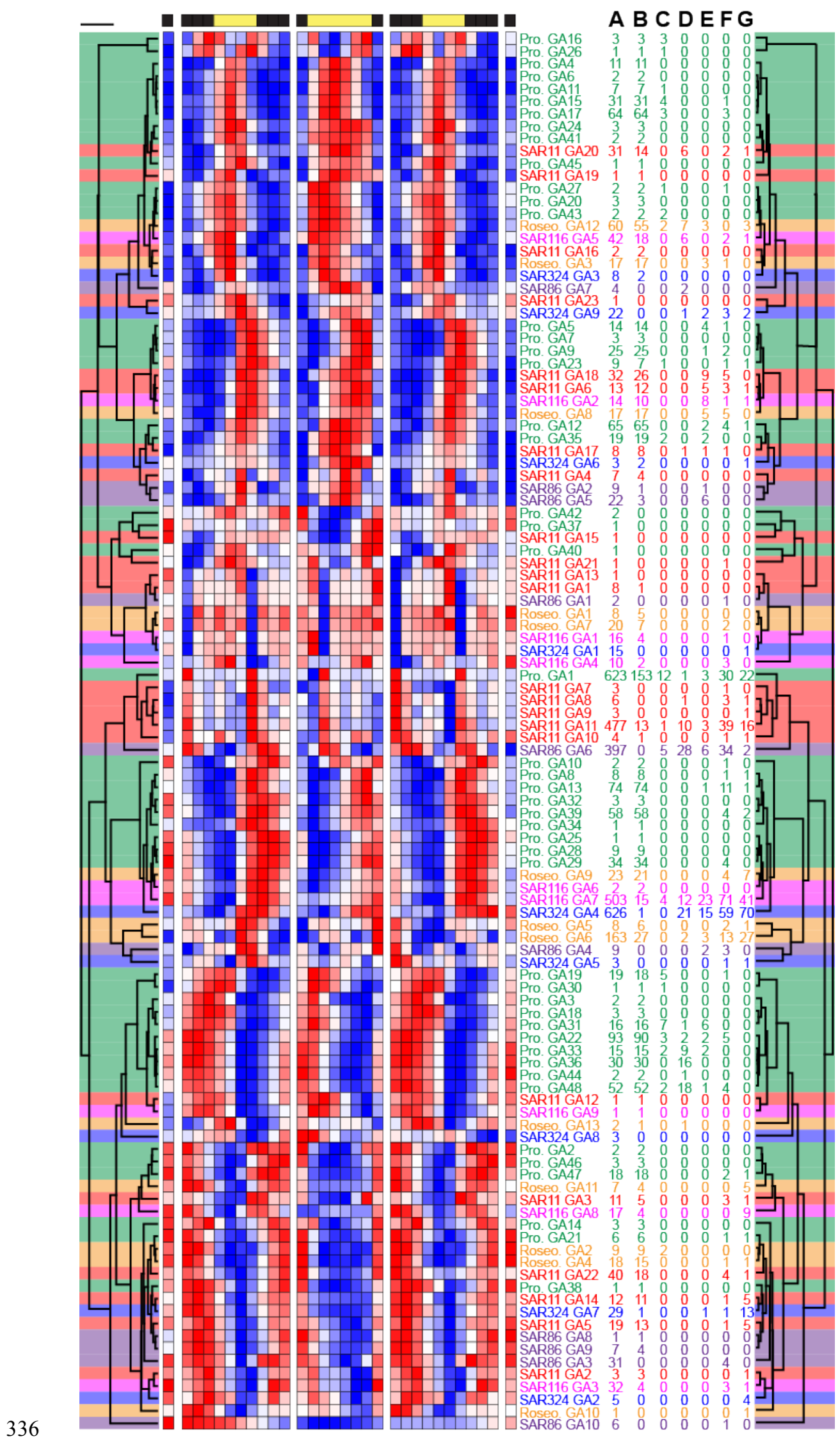

